\title{
Features of photoinduced charge transfer in photomagnetic garnets YIG:Si and YIG:Co
}

\author{
Irina I. Davidenko \\ Kiev Taras Shevchenko National University \\ Volodymyrska Str., 64, 01033, Kiev, UKRAINE \\ (+38044419-81-43, daviden@ukrpack.net)
}

\begin{abstract}
Features of charge transfer under influence of linearly polarized light in photomagnetic garnets YIG:Si and YIG:Co are clarified. Comparative analysis of these two gernets is necessary to create a basis for the theoretical model of photoinduced magnetic effects (PME) in garnets and in other materials as well as for explanation of experimental results of PME observation in YIG:Co even at room temperatures. Known experimental results for YIG:Co are adequately explained and described within the scope of developed simplified theoretical model.
\end{abstract}

Keywords: photoinduced magnetic effect, garnet, impurity center, charge transfer.

Paper received 12.03.01; revised manuscript received 16.05.01; accepted for publication 13.07.01.

\section{Introduction}

The essence of photoinduced magnetic effects (PME) consists in a change of magnetic properties under influence of optical illumination. Physical properties of photomagnetic materials open potential possibilities of their application as a registering medium for the magnetic polarization holography. Amongst these properties, the most important one is the sensitivity of the medium to a polarization of initiating light.

In reality, all known PME could be divided into two classes: the independent on light polarization and polarization sensitive ones [1]. The former include changes of magnetic susceptibility and cubic magnetic anisotropy happening under influence of unpolarized light. Photoinduced uniaxial magnetic anisotropy, photoinduced optical dichroism, spin-reorientation transitions (SRT), transitions between antiferromagnetic and metamagnetic states within the sample region illuminated with linearly polarized light appear among the polarization sensitive PME. From the viewpoint of application possibilities, one of the most interesting effects is the spin-orientation one. It consists in appearance of a magnetic heterogeneity profile under the influence of optical illumination. Parameters of such homogeneities are determined by the light polarization, intensity and geometric characteristics of light spot. Formed in such a way distribution of magnetization is usually visualized using the Faraday effect.

Earlier the most of PME in different materials were observed at cryogenic temperatures. Only recently photoinduced SRT was discovered within the wide temperature range (from liquid helium to room temperature) in epitaxial films of Co-doped YIG (YIG:Co) [2,3]. This determines an additional interest to possibilities of materials with photomagnetic properties for applications as media for information storage. It is obvious that the possibility of photoinduced creation of heterogeneities of magnetization both in thin epitaxial films and in bulk crystals [4] is of great importance.

The present work is aimed to ascertain the features of charge transfer under influence of linearly polarized light in photomagnetic garnets YIG:Si and YIG:Co. Such consideration are necessary to create a basis for the theoretical model of PME in garnets and in other materials and for explanation of experimental results of PME observation in YIG:Co even at room temperatures.

\section{Theory and discussion}

When interpreting the physical nature of PME, it should be taken into account that optical irradiation affecting on the electron impurity subsystem, can change both exchange and relativistic interactions in the magnetic sub- 


\section{I.I. Davidenko: Features of photoinduced charge transfer in photomagnetic ...}

system. The general condition of PME appearance is a presence of highly anisotropic light sensitive impurity centers in the crystal lattice. A role of such centers is played by anisotropic magnetic ions able to exist in states with different valencies. This circumstance and polarization dependency of the whole class of PME testify that relativistic mechanism is more probable. In accordance to modern conception, the nature of PME is connected with optical excitation of anisotropic impurity centers and redistribution of their occupancies between nonequivalent crystal sites $[1,3]$. Such redistribution shows itself in appearance of macroscopic photoinduced magnetic anisotropy (PMA) under influence of polarized optical irradiation.

Within the scope of simplified microscopic model of PMA [5] the formation of anisotropic light sensitive impurity centers in YIG:Si and redistribution of their populations under influence of linearly polarized light was explained in the following manner. Presence of $\mathrm{Si}^{4+}$ ions instead of $\mathrm{Fe}^{3+}$ ions in tetrahedral sites of garnet lattice provides an «extra» electron appearance. To preserve charge neutrality donor electrons transform octahedral ions $\mathrm{Fe}^{3+}$ into highly anisotropic ions $\mathrm{Fe}^{2+}$. It results in disturbance of initial cubic symmetry of garnet matrix. If donor electron is localized on octahedral $\mathrm{Fe}^{2+}$ ion, then local symmetry of ligand environment arises with axes along crystallographic direction $<111>$. Four types of such orientation for non-equivalent octahedral sites appear with local axis of symmetry along directions $<111>_{i}(i=1,2,3,4)$. Linearly polarized light removes degeneracy in populations of impurity centers with electrons, which exists before the illumination. As a result, axes of magnetic and/or optical anisotropy appear.

Nature of anisotropic impurity centers in YIG:Co could be considered in the same way. It is known that in many kinds of ferrites even relatively small cobalt amount involves drastic changes of the anisotropy due to orbital degeneracy of its ground state in threefold field of octahedral sites in spinels and garnets [6]. That is why, it would be natural to expect that PME in Co-substituted garnets should have greater magnitude, as compared to YIG:Si, and slower relaxation under temperature growth.

It is evident that the distinction of the temperature ranges of PME existence in early investigated pure YIG [7], YIG with divalent (YIG:Ca) [8] and tetravalent (YIG:Si) [1,5] dopants from YIG:Co is a consequence of different physical nature and properties of light sensitive anisotropic impurity centers which are responsible for PME. It allows to assume that existence of high temperature polarization dependent PME in YIG:Co is determined just by presence of cobalt ions in the garnet matrix. A symmetry of shown in Fig. 1 dependency of PME time on light polarization [9] testifies that light sensitive centers occupy the octahedral sites in the crystal lattice because minim positions correspond to polarization directions where a probability of photoexcitation of octahedral centers is the largest. It is known $[10,11]$ that $\mathrm{Co}^{2+}$, $\mathrm{Co}^{3+}$ and $\mathrm{Fe}^{3+}$ ions could be found in these positions. Octahedral $\mathrm{Co}^{3+}$ gives a zero contribution into total

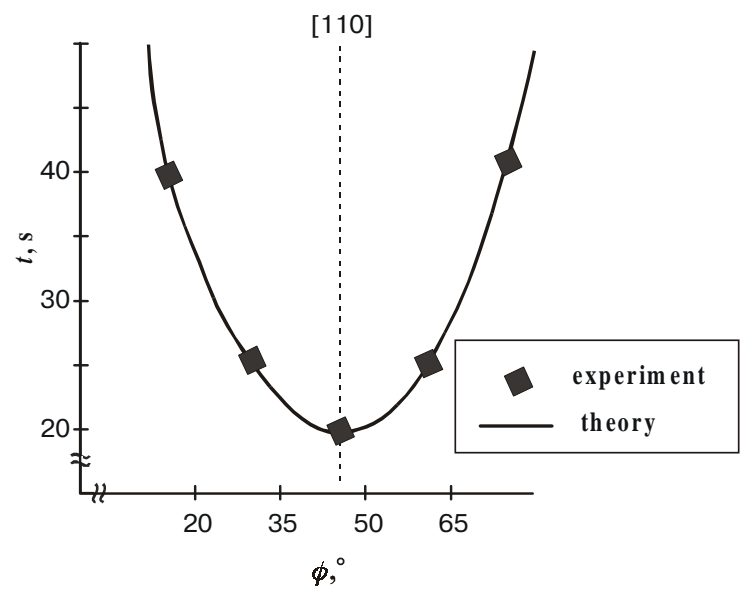

Fig. 1. The dependency of SRT time in YIG:Co on the direction of the incident light polarization.

anisotropy in single ion approximation, and $\mathrm{Fe}^{3+}$ has a much smaller contribution as compared with $\mathrm{Co}^{2+}[11]$. Therefore, redistribution of these ions under light influence could not involve observed PME. Thus, appearance of $\mathrm{PME}$ and respective magnetization changes are connected with redistribution of $\mathrm{Co}^{2+}$ ions amongst the octahedral sites. This redistribution is caused by the optical recharge as well as thermoactivated processes.

Optical recharge accomplishes through an absorption of light quantum with proper polarization in initial octahedral $\mathrm{Co}^{2+}$, ion excitation and electron transition from $\mathrm{Co}^{2+}$ ion to $\mathrm{Co}^{3+}$ ion in another site converting it into $\mathrm{Co}^{2+}$. Such optical recharge provides preferred occupancy for the sites with local symmetry axes $<111\rangle_{i}$ closest to the direction of light polarization due to existence of anisotropy in probability of photoabsorption.

At final temperature of the sample the thermoactivated process always exists resulting in fact that $\mathrm{Co}^{2+}$ could appear again in initial octahedral site. The thermoactivated process provides isotropic redistribution of occupancies amongst all four types of sites with symmetry axis $<111\rangle_{i}$.

The thermoactivated electron transitions between centers are the reason of limitation of temperature range where PMEs exist. Unbalance of populations of impurity centers in YIG:Si induced with linearly polarized light fails at temperature rise starting from $T \approx 120 \mathrm{~K}$ and disappears at all when the critical temperature $T=134 \mathrm{~K}$ [12] is exceeded.

An analysis of the temperature dependencies of the characteristic times of SRT in YIG:Co (curves 1 and $1^{\prime}$ in Fig. 2) [2] and comparison with respective results for YIG:Si (curves 2 and 2' in Fig. 2) [12] permit to more exactly ascertain the microscopic mechanism of PME in YIG:Co. Unfortunately, these graphs present unique known and published results of measurements of the temperature dependencies of PME in these materials. Naturally, this problem needs more precise and detailed consideration. However, in our investigations the fundamental character of these dependencies is important. It ena- 


\section{I.I. Davidenko: Features of photoinduced charge transfer in photomagnetic ...}

bles to make some conclusions about a mechanism of photoinduced electron transitions between the light sensitive centers as well as about their physical nature.

Dependencies in Fig. 2 demonstrate an essential difference between the temperature ranges of PME existence in YIG:Si and YIG:Co. Extrapolation of these experimentally measured dependencies in terms of $\ln t(1 / T)$ by linear dependencies (see insertion in Fig. 2) testifies their exponential character within the investigated temperature ranges with the following characteristic times:

$$
\begin{aligned}
& \tau^{(\mathrm{Si})}=\tau_{0}^{(\mathrm{Si})} \exp \left[-\varepsilon^{(\mathrm{Si})} / \mathrm{kT}\right] \\
& \tau^{(\mathrm{Co})}=\tau_{0}^{(\mathrm{Co})} \exp \left[-\varepsilon^{(\mathrm{Co})} / \mathrm{kT}\right]
\end{aligned}
$$

where $\varepsilon^{(\mathrm{Si})}$ and $\varepsilon^{(\mathrm{Co})}$ are the activation energies of $\tau^{(\mathrm{Si})}$ and $\tau^{(\mathrm{Co})}$ respectively. The values $\varepsilon^{(\mathrm{Si})}$ and $\varepsilon^{(\mathrm{Co})}$ where calculated from the inclination angles of the linear dependencies extrapolating dependencies $\ln t(1 / T): \varepsilon^{(\mathrm{Si})} \approx$ $\approx \varepsilon^{(\mathrm{Co})}=0.071 \pm 0.005 \mathrm{eV}$.

The possibility to present these characteristic time in forms (1a)-(1b) is an evidence of the band character of the electron transitions between centers. The similar conclusion for YIG:Si was made earlier [13]. Exponential dependencies (1a)-(1b) are typical for processes in which a probability of carrier transitions from the photogeneration center into new localized state decreases with temperature growth, and the opposite processes are difficult due to some reasons or poorly exhibited. Such processes are similar to electronic transitions through conductivity band in impurity semiconductors, which are limited by a scattering on phonons within considered temperature range.

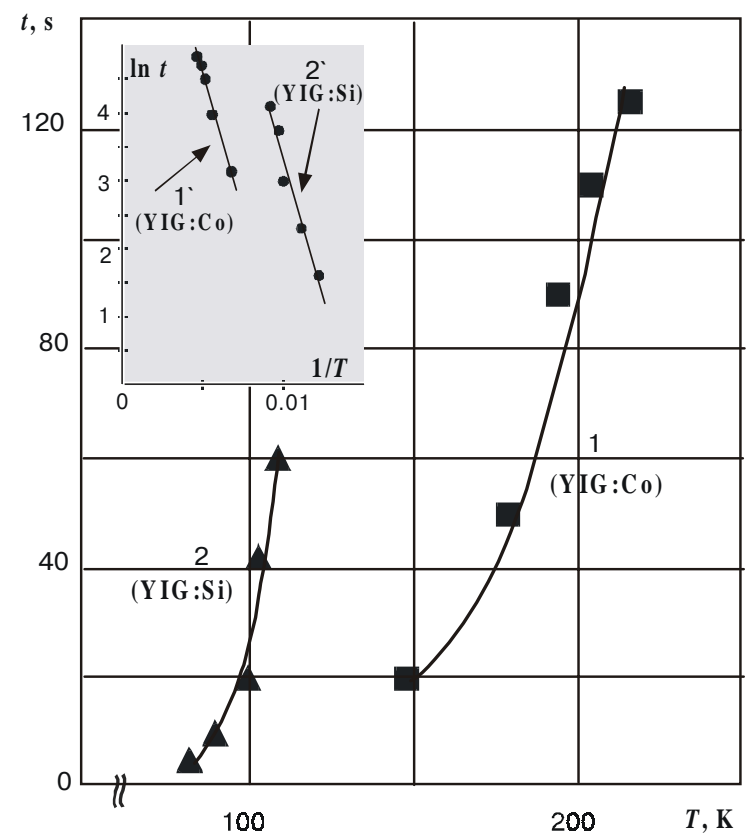

Fig. 2. Temperature dependencies of the saturation time of SRT in YIG:Co [2] and in YIG:Si [12]
An approximate equality of the activation energies $\varepsilon^{(\mathrm{Si})}$ and $\varepsilon^{(\mathrm{Co})}$ seems to be of interest. Apparently, it is an evidence of identity of the photoinduced electronic transitions between active centers in these materials, namely their band character with scattering on optical phonons having energy $\approx 0.1 \mathrm{eV} \mathrm{[14]} \mathrm{in} \mathrm{YIG.} \mathrm{A} \mathrm{difference} \mathrm{between}$ the temperature ranges of PME appearance in considered materials follows from the different nature of active centers. The energetic level of the octahedral ion $\mathrm{Co}^{2+}$ in the ground state is situated $\approx 1 \mathrm{eV}$ below an edge of the conductivity band [15] formed by overlapping 3d-orbitals of iron ions. This makes it difficult for thermoactivated electron to come out from $\mathrm{Co}^{2+}$ and prevents its motion between anisotropic active centers through conductivity band, contrary to $\mathrm{Fe}^{2+}$ ions in YOG:Si. It, in its turn, hampers the relaxation of the non-equilibrium photoinduced distribution of populations into the initial state under temperature growth without light. The fact that for YIG:Si excitation IR light was used $(\lambda=1.15 \mu \mathrm{m})$ [12], whereas in YIG:Co much higher energies $(\lambda=0.488 \mu \mathrm{m})$ $[2,3]$ are required also confirms a regularity of such statement.

The conducted analysis enables to describe the microscopic mechanism of appearance of photoinduced magnetic anisotropy in the photomagnetic materials YIG:Si and YIG:Co under the influence of linearly polarized light. Formally, we could use known [14] model of four types of orientationally non-equivalent octahedral sites. Earlier this model was utilized for description of thermal annealing in a case of localized anisotropic ions. In our case, not ionic transitions between lattice sites, but electronic transitions between photoexcited anisotropic ions in these sites should be taken into account.

Now we can consider in detail the process of photoinduced redistribution of occupancies of octahedral centers in YIG:Si and analyze its difference from that in YIG:Co. Virtually, this process could be presented as consisting of two processes: i) photogeneration and transport of electron within the photomagnetic crystal until a capture on $\mathrm{Fe}^{3+}$ accompanying by appearance of highly anisotropic ion $\mathrm{Fe}^{2+}$; ii) thermoactivated process with returning electron to the initial state. Accordingly, a velocity of the process can be presented by the following expression:

$\omega_{i}=K I v_{i}+v \exp \left[-\varepsilon^{(r)} / k T\right]$

where the first and the second terms characterize optical recharge and thermal relaxation, respectively. Index $i$ denotes the type of the octahedral site, i.e. orientation of its trigonal axes along one of $\langle 111\rangle_{i}$ directions. $I$ is the light intensity, $K$ is the probability of electron transition from excited state of center, where the light quantum was absorbed, into another center, $v$ is the frequency factor, $\varepsilon^{(r)}$ is the activation energy of thermoactivated electron transition, $v_{\mathrm{i}}=A\left(1+B \cos ^{2} \varphi_{i}\right)\left(1+C \cos ^{2} \psi_{i}\right)$ is the anisotropic probability of absorption of light quantum with unit intensity, $A, B$ and $C$ are the phenomenological constants, $\varphi_{i}$ and $\psi_{i}$ are the angles between local symmetry axes of $i$-type site $\left.(<111\rangle_{i}\right)$ and directions of light polarization $\vec{E}$ and magnetization $\vec{M}$, respectively. 


\section{I.I. Davidenko: Features of photoinduced charge transfer in photomagnetic ...}

Expressions similar (2) earlier were used for description of the thermal annealing [14] and photoinduced processes [5] in YIG:Si without detailed explanation of the probability $K$ which depends on temperature accordingly to expression (1a). Transport of charge carriers in YIG:Co accomplishes through energetic band formed by iron ions. It can serve as formal foundation for using the model described above with some modification of the expression (2):

$\omega_{\mathrm{i}}^{(\mathrm{Co})}=g I A\left(1+B \cos ^{2} \varphi_{\mathrm{i}}\right)\left(1+C \cos ^{2} \psi_{\mathrm{i}}\right) v_{1}$

$\exp \left[\varepsilon_{1}^{(\mathrm{Co})} / k T\right]+n_{2} \exp \left[-\varepsilon_{2}(\mathrm{Co}) / k T\right]$

where $g$ is the probability of $\mathrm{Co}^{2+}$ excitation and electron coming out into conductivity band, $v_{1}$ is the frequency factor of motion of delocalized electron and its transition into free energetic level of $\mathrm{Co}^{3+}$ ion in neighbor site, $\varepsilon_{1}(\mathrm{Co})$ is the activation energy of the electronic mobility within the conductivity band depending on electronphonon interaction, $v_{2}$ and $\varepsilon_{2}{ }^{(\mathrm{Co})}$ are the frequency factor and activation energy of thermoactivated electron transition from $\mathrm{Co}^{2+}$ ion into conductivity band, respectively.

Evidently, the dependency on $\varphi_{i}$ and $\psi_{i}$ in the expression (3) determines different velocities of changes of concentration in the center of given type corresponding to the orientation of its local symmetry axes respectively to the light polarization and magnetization, i.e. the strong polarization dependency of SRT takes place. Shown in Fig. 1 dependency of the SRT time on direction of light polarization testifies to it.

Within the scope of developed simplified model the photoinduced redistribution of site occupancies have been described by the system of kinetic equations with equation of conservation of total number of centers:

$$
\left\{\begin{array}{l}
\frac{d n_{i}}{d t}=-\omega_{i} n_{i}+\frac{1}{3} \sum_{j=1}^{3} \omega_{j} n_{j}, i \neq j . \\
\sum_{i=1}^{4} n_{i}=N
\end{array}\right.
$$

where $n_{i}$ are the concentrations of centers of $i$-type, $N$ is the total concentration of octahedral $\mathrm{Co}^{2+}$ ions.

Numerical simulation within the scope of the developed model and calculating of the energy of PMA accordingly to Slonczewski's model enable to make theoretical fitting of the experimental dependencies in Fig. 1. Achieved results are plotted by the solid line.

\section{Conclusions}

Thus, basing on the analysis of the available at the present time experimental results of PME investigations in YIG with different dopants, the simple microscopic model for the description of polarization sensitive PME in YIG:Co within wide temperature range was developed. Experimental results were explained and quite adequately described within the scope of the model. As it follows from the conducted analysis, the reason of existence of PME in YIG:Co at more high temperatures, as compared with YIG:Si, is connected with difference in properties of anisotropic impurity centers responsible for PME as well as with peculiarities of charge transfer between these centers in different materials.

Developed model could be generalized and used for other media with photomagnetic properties taking into account nature of photoactive light sensitive centers responsible for PME and features of photoinduced charge transfer between these ones.

The researches were financially supported within the scope of the project INTAS 97-0366.

\section{References}

1. V.F. Kovalenko and E.L. Nagaev// Sov.Phys.Usp., 29, 297 (1986); E.L. Nagaev, Phys.Status Solidi, 145, 11 (1988).

2. A.B. Chizhik, S.N. Lyakhimets, A. Maziewski, M. Tekielak // JMMM, 140-144, 2111 (1995).

3. A.B. Chizhik, I.I. Davidenko, A. Maziewski, A. Stupakiewicz // Phys.Rev.B., 57, 22 (1998).

4. I.I. Davidenko, F.F. Kovalenko, Proc. of Intern. Conf. European Magnetic Materials \& Applications, Kosice, 1993, P.298.

5. V.F. Kovalenko, P.S. Kuts, S.N. Lyakhimets// FTT, 24, 2428 (1982).

6. J.C. Slonczewski // Phys.Rev., 110, 1341 (1958).

7. V.G. Veselago, R.A. Doroshenko, S.G.Rudov // Zh. Exp. Teor. Fiz., 105, 638 (1994).

8. M. Pardavi-Horvath, P.E. Wigen, G.Vertesy // J.Appl.Phys., 63, 3110 (1988)

9. I.I. Davidenko, A. Stupakiewics, A.L. Sukstanskii, A. Maziewski // FTT, 39, 1824 (1997).

10. B. Antonini, M. Marinelli, E. Milani, A. Paoletti, P. Parolli, B. Ferrand // Phys.Rev.B., 39, 13422 (1989).

11. M.D. Sturge, E.M. Gyorgy, R.C. LeCraw, J.P. Remeika // Phys.Rev., 180, 413 (1969).

12. P.S. Kuts, V.V. Ionov, V.P. Sokhatski, S.N. Kyakhimets, V.F. Kovalenko // Izv.VUZ, Physics, 66 (1986).

13. K. Hisatake, S.N. Lyakhimets, Proc.of Intern.Conf. on Ferrites, Tokyo, 1535 (1993).

14. R.P. Hunt // J.Appl.Phys., 38, 2826 (1967).

15. G.H.Jonker // J.Phys.Chem.Sol., 9, 325 (1959). 